\title{
IUWDS International Geophysical Calendar for 1970
}

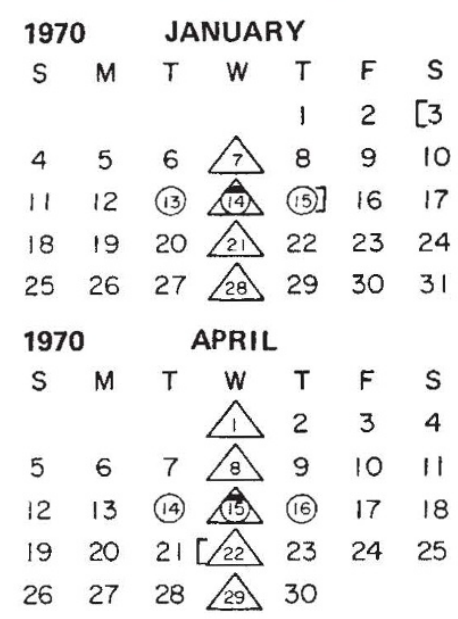

1970 JULY

$S \quad M \quad T \quad W \quad T \quad F \quad S$

$\bigwedge \begin{array}{llll}1 & 2 & 3 & 4\end{array}$

$\begin{array}{lllllll}5 & 6 & 7 & \text { 8 } & 9 & 10 & 11\end{array}$

$\begin{array}{llllll}12 & 13 & \text { (14) (15) (16) } 17 \quad 18\end{array}$

$\begin{array}{lllllll}19 & 20 & 21 & 222 & 23 & 24 & 25\end{array}$

$\begin{array}{ll}26 & 27][28][29][30] 31]\end{array}$

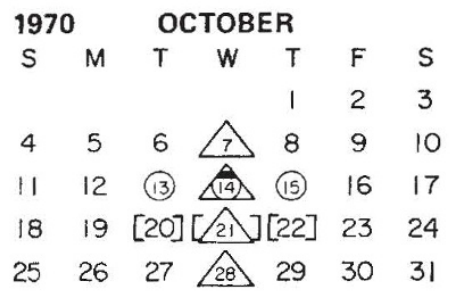

1971 JANUARY

$S$ S $T$ W $T$ T $F$

$\begin{array}{llllllll}3 & 4 & 5 & 6 & 7 & 8 & 9\end{array}$

$\begin{array}{lllllll}10 & 11 & \text { (12) } & \text { (13) (14) } 15] \quad 16\end{array}$

$\begin{array}{lllllll}17 & 18 & 19 & 20 & 21 & 22 & 23\end{array}$

$\begin{array}{lllllll}24 & 25 & 26 & 27 & 28 & 29 & 30\end{array}$

\begin{tabular}{|c|c|c|c|c|c|}
\hline \multicolumn{2}{|c|}{770} & \multicolumn{3}{|c|}{ FEBRUARY } & \\
\hline 5 & $M$ & T & w & $T$ & $\mathrm{~F}$ \\
\hline & 2 & 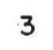 & $\Delta$ & 5 & 6 \\
\hline & 9 & (1) & (Ai) & (12) & 13 \\
\hline & 16 & 17 & & 19 & 20 \\
\hline & 23 & 24 & & 26 & 27 \\
\hline
\end{tabular}

1970 MAY

$\begin{array}{lllllll}S & M & T & W & T & F & S\end{array}$

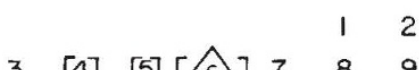

$\begin{array}{lllllll}10 & 11 & \text { (12) (13) (14) } 15 \quad 16\end{array}$

$\begin{array}{lllllll}17 & 18 & 19 & 20 & 21 & 22 & 23\end{array}$

$\begin{array}{lllllll}24 & 25 & 26 & 202 & 28 & 29 & 30\end{array}$

31

1970 AUGUST

$S \quad M \quad T \quad W \quad T \quad F \quad S$

53407

$9\left[10 \quad[1 1 \longdiv { 1 2 }]\left[\begin{array}{lll}13 & {[14} & 15\end{array}\right.\right.$

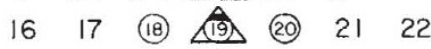

$\begin{array}{lllllll}23 & 24 & 25 & 26 & 27 & 28 & 29\end{array}$

30 31-

1970 NOVEMBER

$\begin{array}{lllllll}S & M & T & W & T & F & S\end{array}$

$123 \triangle 4567$

$\begin{array}{lllllll}8 & 9 & 10 & \text { 11 } & 12 & 13 & 14\end{array}$

15 [16] [117] $\quad 180$ (19) $20 \quad 21$

$\begin{array}{lllllll}22 & 23 & 24 & 25 & 26 & 27 & 28\end{array}$

2930

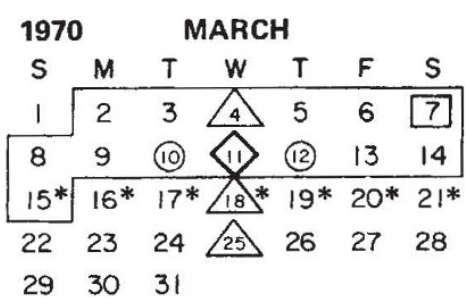

1970 JUNE

S $M \quad T \quad W \quad T \quad F \quad S$

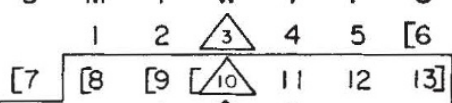

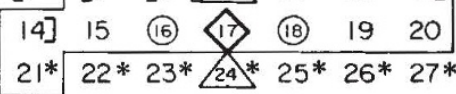

$28 \quad 29 \quad 30$

\section{SEPTEMBER}

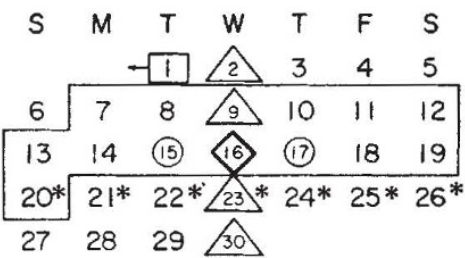

1970 DECEMBER

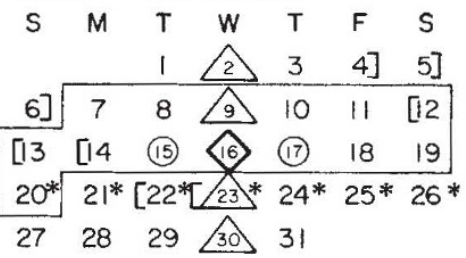

(14) Regular World Doy (RWD)

(13) Priority Regular World Doy (PRWD)

(16) Quarterly World Day (QWD) also a PRWD and RGD

12. Regular Geophysical Day (RGD)

5 Day of Solar Eclipse

$6 \quad 7$ World Geophysical Interval (WGI)
[2 Day with unusual meteor shower activity,

Northern Hemisphere

3] Day with unusual meteor shower activity,

Southern Hemisphere

* Micropulsation Interval Day

TABLE OF WORLD DAYS MARKED ON THE CALENDAR

\begin{tabular}{|c|c|c|c|c|c|c|c|c|}
\hline 1970 & RWD & PRWD & OWD & RGD & WGI & ECL. & METEORS & MICROPULSATION INTERVALS \\
\hline Jan. & $13,14,15$ & 14 & & $7,14,21,28$ & & & 3,15 & \\
\hline Feb. & $10,11,12$ & 11 & & $4,11,18,25$ & & & & \\
\hline Mar. & $10,11,12$ & 11 & 11 & $4,11,18,25$ & $2 \cdot 15$ & 7 & & $15-21$ \\
\hline Apr. & $14,15,16$ & 15 & & $1,8,15,22,29$ & & & 22 & \\
\hline May & $12,13,14$ & 13 & & $6,13,20,27$ & & & 4-6 & \\
\hline June & $16,17,18$ & 17 & 17 & $3,10,17,24$ & $8 \cdot 21$ & & $6-10,13-14$ & 21.27 \\
\hline July & $14,15,16$ & 15 & & $1,8,15,22,29$ & & & $27-31$ & \\
\hline Aug. & $18,19,20$ & 19 & & $5,12,19,26$ & & $31>$ & $10 \cdot 14$ & \\
\hline Sep. & $15,16,17$ & 16 & 16 & $2,9,16,23,30$ & $7-20$ & $\Rightarrow 1$ & & $20-26$ \\
\hline Oct. & $13,14,15$ & 14 & & $7,14,21,28$ & & & $20-22$ & \\
\hline Nov. & $17,18,19$ & 18 & & $4,11,18,25$ & & & $16-17$ & \\
\hline Dec. & $15,16,17$ & 16 & 16 & $2,9,16,23,30$ & 7.20 & & $4-6,12-14$, & $22-23$ \\
\hline
\end{tabular}

